\title{
A Newly Characterized Hemoglobin Variant with a High Oxygen Affinity, Hb Fuchu-II, Presenting with Acute Myocardial Infarction
}

\author{
Raku Son ${ }^{1}$, Takakazu Higuchi ${ }^{2}$, Atsushi Mizuno ${ }^{3}$, Ryosuke Koyamada ${ }^{2}$, \\ Sadamu Okada ${ }^{2}$ and Yasuhiro Yamashiro ${ }^{4}$
}

\begin{abstract}
A 65-year-old Japanese man presented with acute myocardial infarction (AMI) and polycythemia. Biochemical studies of the patient's hemoglobin $(\mathrm{Hb})$ and the sequencing of his globin genes revealed that the polycythemia was secondary to a high oxygen affinity $\mathrm{Hb}$ variant, $\mathrm{Hb}$ Fuchu-II. Hb variants with high oxygen affinity can be an additional thrombotic risk factor in older patients and/or those with other risk factors. The patient was diagnosed with hemoglobinopathy after the development of AMI and exemplifies the importance of recognizing such conditions and of taking appropriate prophylactic interventions.
\end{abstract}

Key words: hemoglobin variant, high oxygen affinity, Hb Fuchu-II, polycythemia, coronary heart disease

(Intern Med 55: 285-287, 2016)

(DOI: 10.2169/internalmedicine.55.5311)

\section{Introduction}

Approximately 100 hemoglobin $(\mathrm{Hb})$ variants with high oxygen affinity have been identified which may lead to secondary polycythemia (1). While several prospective and cohort studies and a meta-analysis have suggested that increased hematocrit $(\mathrm{Ht})$ and $\mathrm{Hb}$ levels are associated with increased risk of cardiovascular events (2) and ischemic heart disease (IHD) (3-5), young patients with polycythemia secondary to $\mathrm{Hb}$ variants with high oxygen affinity usually follow a benign clinical course and need no treatment. However, polycythemia secondary to $\mathrm{Hb}$ variants is often associated with thrombotic events in older patients and/or those with other risk factors (1).

We herein report the case of a patient presenting with acute myocardial infarction (AMI) and polycythemia in whom the polycythemia was proven to be secondary to a newly characterized high oxygen affinity $\mathrm{Hb}$ variant, $\mathrm{Hb}$ Fuchu-II, and was considered to have contributed to the development of AMI as an additional cardiovascular risk factor.

\section{Case Report}

A 65-year-old Japanese man presented to our emergency department with a chief complaint of chest pain, which had started suddenly one hour before and radiated to the shoulders. He reported that arrhythmia had once been identified in an annual health checkup but that it had not been further evaluated. He had a brother who had previously undergone coronary angiography (CAG). He was a current smoker and smoked 10-15 cigarettes a day.

At presentation, his blood pressure was $96 / 71 \mathrm{mmHg}$. His pulse rate was $74 \mathrm{bpm}$ and was regular in rhythm. With the exception of cold extremities, his physical examinations were unremarkable. An electrocardiogram showed STsegment elevations and hyper acute $\mathrm{T}$ waves in leads V1-V5 and a bedside echocardiogram showed abnormal wall motions in the anterior wall and the apex of the heart. A diagnosis of ST-segment elevation myocardial infarction was made and he was admitted to hospital. The patient's laboratory data showed the following: white blood cell count $6.9 \times$ $10^{9} / \mathrm{L}$, red blood cell (RBC) count $6.08 \times 10^{12} / \mathrm{L}, \mathrm{Hb} 18.3 \mathrm{~g} /$

${ }^{1}$ Internal Medicine, St. Luke's International Hospital, Japan, ${ }^{2}$ Division of Hematology, St. Luke's International Hospital, Japan, ${ }^{3}$ Division of Cardiology, St. Luke's International Hospital, Japan and ${ }^{4}$ Faculty of Medicine and Health Sciences, Yamaguchi University, Japan

Received for publication March 9, 2015; Accepted for publication April 29, 2015

Correspondence to Dr. Takakazu Higuchi, takhig@luke.ac.jp 


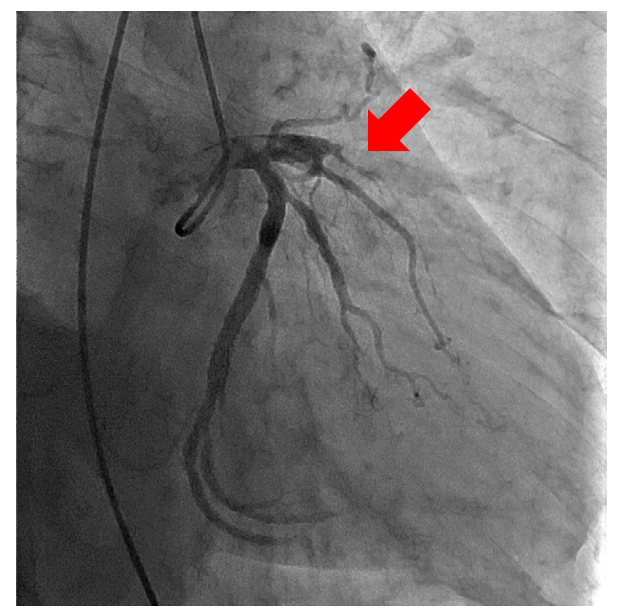

Figure 1. Coronary angiography (CAG) at the onset of acute myocardial infarction. Emergent CAG performed on admission reveals an occlusion of the \#6 branch of the left anterior descending artery (arrow).

$\mathrm{dL}$, Ht $54.8 \%$, and a platelet count of $141 \times 10^{9} / \mathrm{L}$. The results of coagulation studies were normal. Blood chemistry tests revealed the following: creatinine kinase (CK) 115 IU/ L, CK-MB 23 IU/L, glucose $114 \mathrm{mg} / \mathrm{dL}$, and HbA1c 3.9\%. Emergent CAG was performed, which revealed the occlusion of the \#6 branch of the left anterior descending artery (Fig. 1) and a bare metal stent was implanted. The CK and CK-MB peaked at 7,295 IU/L and $675 \mathrm{IU} / \mathrm{L}$, respectively. Treatment with low-dose aspirin and clopidogrel was started. Warfarinization was performed due to the identification of a left ventricular thrombus on a transthoracic echocardiogram, and he was discharged on the 11th day after admission.

After discharge, the laboratory data still showed erythrocytosis with an $\mathrm{Hb}$ level of $17.7 \mathrm{~g} / \mathrm{dL}$, an Ht level of $53.9 \%$, and an HbAlc level of $4.4 \%$, which was low, and raised the possibility of hemoglobinopathy. He reported that polycythemia had been repeatedly pointed out on annual checkups and that his brother also had polycythemia. Additional laboratory data revealed a total bilirubin level of $0.7 \mathrm{mg} / \mathrm{dL}$, a lactate dehydrogenase level of $191 \mathrm{IU} / \mathrm{mL}$ (reference range, 118-223), and a haptoglobin level of $73 \mathrm{mg} / \mathrm{dL}$. The serum erythropoietin level was mildly elevated at $56.2 \mathrm{mIU} / \mathrm{mL}$ (reference range, 9.1-32.8). A peripheral blood smear revealed no morphological abnormalities of the RBCs. The isoelectrofocusing of the RBC lysate showed an abnormal band overlapping HbA1c. The HbAlc content was determined by a routine high performance liquid chromatography (HPLC) analysis to be $4.4 \%$, and no abnormal peaks were detected. The sequencing of the globin genes revealed a substitution of $\mathrm{AAC}(\mathrm{Asn}) \rightarrow \mathrm{CAC}(\mathrm{His})$ at codon 97 of the $\alpha$ globin gene, or $\mathrm{Hb}$ Fuchu-II (6). The $\alpha 1$ - and $\beta$-globin genes were normal. A gap-polymerase chain reaction (PCR) unexpectedly revealed a -3.7 type deletion in the $\alpha$-globin gene $\left(-\alpha^{3.7}\right)$, however, the glycerol lysis time was not prolonged and $\mathrm{HbH}$ inclusion bodies were not observed. The oxygen dissociation curve of the patient's whole hemolysate

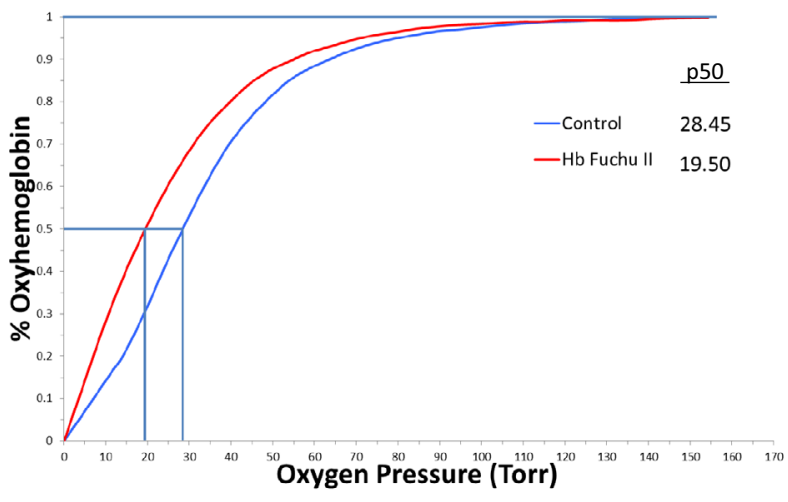

Figure 2. The oxygen equilibrium curve of the hemoglobin (Hb). The oxygen dissociation curve of the patient's Hb (red curve) was shifted to the left compared to the control (blue curve) and the p50 was 19.5 Torr, compared to 28.5 Torr in the control.

was shifted to the left and the p50 was 19.50 Torr, which was lower than that of the control (28.45 Torr) (Fig. 2), thus indicating the high oxygen affinity of $\mathrm{Hb}$ Fuchu-II. When the $\mathrm{O}_{2}$ pressure of mixed venous blood was presumed to be 40 Torr, the decreased A-V difference of his blood (18.2\% vs. $28 \%$ for normal control) suggested that Hb Fuchu-II had a reduced oxygen-carrying capacity. The decreased Hill's coefficient ( 2.28 vs. 2.58 for normal control) suggested a decreased heme-heme interaction or lack of cooperativity during $\mathrm{O}_{2}$ binding and release. The Bohr effect was also decreased ( 0.49 vs. 0.62 for normal control). He currently remains on low-dose aspirin and warfarin treatment and is stable without any symptoms or complications.

\section{Discussion}

While secondary polycythemia usually occurs as a result of an inappropriately increased level of erythropoietin due to tissue hypoxia of various causes and, less frequently, the inappropriate production of erythropoietin, it may occur secondarily to the increased oxygen affinity of $\mathrm{Hb}$. Thus far, approximately $100 \mathrm{Hb}$ variants with high oxygen affinity have been identified (1). $\mathrm{HbA}$ is the major adult $\mathrm{Hb}$ and is a tetramer formed by two identical dimers of $\alpha 1 \beta 1$ and $\alpha 2 \beta 2$. The most frequent alterations causing high oxygen affinity $\mathrm{Hb}$ variants are located either in the $\alpha 1 \beta 2$ interface or at the C-terminal (1). Hb Fuchu-II is an $\alpha$-globin variant $\mathrm{Hb}$ that was identified in an asymptomatic Japanese man with polycythemia and which was first reported in 1995 (6). Hb Fuchu-II has an Asn $\rightarrow$ His substitution at codon 97 of the $\alpha$ globin, which is located in the $\alpha 1 \beta 1$ contact region. Theoretically, mutations occurring in this region could be predicted to affect the molecular stabilization and result in an increased oxygen affinity. However, the biochemical properties of $\mathrm{Hb}$ Fuchu-II have not previously been studied. We studied the oxygen equilibrium curve and the A-V difference of the $\mathrm{Hb}$ of the patient and confirmed that $\mathrm{Hb}$ FuchuII does indeed have a high oxygen affinity and a low 
oxygen-carrying capacity. On the other hand, it is expected that the degree of such phenomena depends on the amount of the high-affinity $\mathrm{Hb}$ variant. Unfortunately, since $\mathrm{Hb}$ Fuchu-II was not separated by isoelectrofocusing, we did not determine the level of $\mathrm{Hb}$ Fuchu-II, while the level in the first case was reported to $25 \%$, as determined by cation exchange HPLC (6). The present case unexpectedly had $\alpha^{+}-$ thalassemia ( $-\alpha^{3.7}$ type) allele, and the Hb Fuchu-II gene was on the other chromosome. Since two $\alpha$-globin genes reside adjacent to each other on chromosome $16 \mathrm{p}$, the $\alpha$-globin genotype of the present case was considered to be $[\alpha-/$ $\left.\alpha \alpha^{\text {Fuchu-II }}\right]$, rather than $\left[\alpha \alpha / \alpha \alpha^{\text {Fuchu-II }}\right]$, with a deletion of one of the four $\alpha$-globin genes. Thus, the content of $\mathrm{Hb}$ Fuchu-II in the present case can be predicted to be a little more than $25 \%$, which indicates that an $\mathrm{Hb}$ Fuchu-II level of more than $25 \%$ is sufficient to cause polycythemia.

The elevation of Ht increases blood viscosity at low shear rates and displaces platelets toward the vessel wall at high shear-rates, facilitating shear-induced platelet activation, and, thus increasing both venous and arterial thrombotic risks (7). It is reported that an $\mathrm{Ht}$ level of above $45 \%$ is associated with a higher rate of cardiovascular death and major thrombosis in patients with polycythemia vera (8). Likewise, although cohort or prospective studies do not always report consistent statistically significant results, possibly due to the study design $(9,10)$, patients with polycythemia due to other etiologies are at an increased risk of IHD (3-5). Therefore, we believe that polycythemia secondary to $\mathrm{Hb}$ FuchuII contributed to the development of AMI in the present patient who had other preexisting cardiovascular risk factors, namely smoking and aging, and also to the formation of left ventricular thrombus. While young patients with polycythemia secondary to high oxygen affinity variant $\mathrm{Hb}$ usually follow a benign clinical course, it is associated with thrombotic events in older patients and/or those with other risk factors (1). Therefore, it is possible that his cardiovascular risk was further raised by the increased Ht level caused by secondary polycythemia. In addition, although we could not confirm the long-term variation of the Ht level in the present case, secondary polycythemia may emerge or progress later in life in patients with certain high oxygen affinity $\mathrm{Hb}$ variants and such patients may be at a higher risk of thrombotic complications as they age (11).

Hemoglobinopathy with high oxygen affinity is assumed to be underdiagnosed due to a lack of awareness about the condition and a lack of access to confirmatory tests. In fact, polycythemia had been repeatedly pointed out without further evaluation in the present case many years before the onset of AMI, which could have been prevented if the diagnosis had been made earlier and appropriate prophylactic measures had been taken. In the present case, the patient's presentation with AMI, and his polycythemia and low HbA1c level prompted us to suspect hemoglobinopathy, illustrating the importance of the appropriate evaluation of these laboratory data.

In conclusion, the present case indicates that we should always consider the possibility of hemoglobinopathy when we see a patient with polycythemia and/or a low HbA1c level since it can be an additional cardiovascular risk, especially in patients with other risks. We should also consider that a diagnosis of hemoglobinopathy may indicate the need to start prophylactic intervention.

The authors state that they have no Conflict of Interest (COI).

\section{Acknowledgement}

We thank Dr. Yukio Hattori for his critical reading of the manuscript.

\section{References}

1. Wajcman H, Galactéros F. Hemoglobins with high oxygen affinity leading to erythrocytosis. New variants and new concepts. Hemoglobin 29: 91-106, 2005.

2. Chonchol M, Nielson C. Hemoglobin levels and coronary artery disease. Am Heart J 155: 494-498, 2008.

3. Wannamethee G, Shaper AG, Wincup PH. Ischaemic heart disease: association with haematocrit in the British Regional Heart Study. J Epidemiol Community Health 48: 112-118, 1994.

4. Kunnas T, Solakivi T, Huuskonen K, et al. Hematocrit and the risk of coronary heart disease mortality in the TAMRISK study, a 28-year follow-up. Preventive Med 49: 45-47, 2009.

5. Danesh J, Collins R, Peto R, Lowe GDO. Haematocrit, viscosity, erythrocyte sedimentation rate: meta-analyses of prospective studies of coronary heart disease. Eur Heart J 21: 515-520, 2000.

6. Harano T, Harano K, Uehara S, Matsushita K. Two new $\alpha$ chain variants: Hb Fuchu-I [ $\alpha 72(\mathrm{EF} 1) \mathrm{His} \rightarrow \mathrm{Tyr}]$ and Hb Fuchu-II $[\alpha 97$ (G4)Asn $\rightarrow$ His]. Hemoglobin 19: 389-395, 1995.

7. Barbui T, Finazzi G, Falanga A. Myeloproliferative neoplasms and thrombosis. Blood 122: 2176-2184, 2013.

8. Marchioli R, Finazzi G, Specchia G, et al. Cardiovascular events and intensity of treatment in polycythemia vera. $\mathrm{N}$ Engl $\mathrm{J}$ Med 368: 22-33, 2013.

9. Gagnon DR, Zhang TJ, Brand FN, Kannel WB. Hematocrit and the risk of cardiovascular disease-The Framingham Study: a 34year follow-up. Am Heart J 127: 674-682, 1994.

10. Lowe GDO, Lee AJ, Rumley A, Price JF, Fowkes FGR. Blood viscosity and risk of cardiovascular events: the Edinburgh Artery Study. Br J Haematol 96: 168-173, 1997.

11. Dash S, Das R. Late emergence of polycythemia in a case of $\mathrm{Hb}$ Chandigarh $[\beta 94(F G 1) A s p \rightarrow$ Gly]. Hemoglobin 28: 273-274, 2001.

(C) 2016 The Japanese Society of Internal Medicine http://www.naika.or.jp/imonline/index.html 